\title{
Innate Phagocyte Polarization in the Oral Cavity
}

\author{
Sarah Metcalfe ${ }^{1+}$, Natalie Anselmi ${ }^{1+}$, Alejandro Escobar ${ }^{2}$, Michelle B. Visser $^{1 *}$ \\ and Jason G. Kay ${ }^{1 *}$ \\ ${ }^{1}$ Department of Oral Biology, School of Dental Medicine, University at Buffalo, Buffalo, NY, United States, ${ }^{2}$ Instituto de \\ Investigación en Ciencias Odontológicas, Facultad de Odontología, Universidad de Chile, Santiago, Chile
}

\section{OPEN ACCESS}

Edited by:

Lesley Ann Bergmeier,

Queen Mary University of London, United Kingdom

Reviewed by:

Antonio Celada,

University of Barcelona, Spain

Felix Ellett,

Massachusetts General Hospital and Harvard Medical School, United States Corneliu Sima,

Harvard School of Dental Medicine, United States

*Correspondence: Jason G. Kay jasonkay@buffalo.edu

Michelle B. Visser mbvisser@buffalo.edu

${ }^{+}$These authors have contributed equally to this work

Specialty section:

This article was submitted to Mucosal Immunity,

a section of the journal

Frontiers in Immunology

Received: 31 August 2021 Accepted: 14 December 2021 Published: 06 January 2022

Citation:

Metcalfe S, Anselmi N,

Escobar A, Visser MB and Kay JG (2022) Innate Phagocyte

Polarization in the Oral Cavity.

Front. Immunol. 12:768479. doi: 10.3389/fimmu.2021.768479
The oral cavity is a complex environment constantly exposed to antigens from food and the oral microbiota. Innate immune cells play an essential role in maintaining health and homeostasis in the oral environment. However, these cells also play a significant role in disease progression. This review will focus on two innate phagocytes in the oral cavity: macrophages and neutrophils, and examine their roles during homeostasis and disease development, with a focus on periodontal disease and cancer. Macrophages have a wellknown ability to polarize and be activated towards a variety of phenotypes. Several studies have found that macrophages' polarization changes can play an essential role in maintaining health in the oral cavity and contribute to disease. Recent data also finds that neutrophils display phenotypic heterogeneity in the oral cavity. In both cases, we focus on what is known about how these cellular changes alter these immune cells' interactions with the oral microbiota, including how such changes can lead to worsening, rather than improving, disease states.

Keywords: macrophage, neutrophil, inflammation, cellular polarization, periodontal disease, oral cancer

\section{INTRODUCTION}

The oral cavity is the main gateway into the human body, leading to the respiratory and gastrointestinal tracts. It has a wide variety of microbial niches, and has the second most abundant microbiota after the gastrointestinal tract, consisting of $\sim 800$ bacterial species categorized into six major phyla (1). In this context, similarly to other mucosal barriers, the local oral immune system needs to find a balance of coexisting with the commensal microbiota while responding appropriately to pathogens (2). The crosstalk between microbiota and the innate immune system is essential to maintaining this host-microbe homeostasis, with the commensal microbiota itself playing a crucial role in regulating immune homeostasis (2-4). Indeed, the oral cavity is a unique mucosal environment where immune cells must be able to recognize and eliminate pathogens while maintaining tolerance to food antigens and the resident microbiota. Oral immunity is composed of a diverse and dynamic network of interactions with both innate and adaptive immunity components contributing to the maintenance, integrity, and host protection of oral tissues. However, as innate immunity is the first line of defense, it plays a pivotal role in both protecting the host and maintaining homeostasis $(5,6)$. While the saliva and gingival crevicular fluid (GCF) contain host immune molecules that can respond rapidly to protect the periodontium and the oral hard tissues against pathogens such as antimicrobial peptides, complement, and secretory 
IgA (7), these defense mechanisms only provide short-term protection and have a limited specificity (8). The oral mucosa resident and transmigrating immunologically active innate immune cells, including macrophages and neutrophils, also play important roles in maintaining effective immune surveillance.

The importance of innate professional phagocytes in maintaining a healthy and mature immune system is revealed upon a change in 'ideal' functional inflammatory immune cell infiltrate: such changes lead to degradation in the health of the periodontal tissues (4). For example, a lack of neutrophil infiltration into the oral cavity (neutropenia) leads to an increase in periodontal disease (9) while an overabundance and dysregulation of neutrophils during periodontal disease causes host tissue damage (10). Similarly, a reduction in macrophage numbers during aging contributes to an increase in periodontal disease (11), while macrophages themselves also contribute to the alveolar bone resorption seen during $P$. gingivalis induced periodontal disease (12). Recent data suggests part of this finely tuned balance of phagocytes is likely due to phenotypic variance within these cell types. Macrophages are responsible for not only host defense, but also have important tissue repair and homeostatic roles (13) and possess a spectrum of phenotypes with different responses during host and microbial interactions (14); (15). Neutrophil phenotypes during infection, inflammation and cancer are also being recognized (16-19), including in response to periodontal disease (20-23). This review will examine the current knowledge of the role polarization, or phenotypic changes, in macrophages and neutrophils is thought to play in the oral environment, especially during the development of periodontal disease.

\section{MACROPHAGE FUNCTIONS IN THE ORAL ENVIRONMENT}

Macrophages are located in the lamina propria below the epithelium and are among the first innate cells to interact with microorganisms and microbial products, and so are an important cell type under both homeostatic and disease conditions in the oral cavity. Under physiological conditions macrophages are important for cell turnover and maintenance of the extracellular milieu (24), while also being required to recognize, internalize and kill microbes in order to clear infections (25). The recognition of microbes by macrophages also results in production of proinflammatory cytokines, which contribute to inflammation initiation (26). Moreover, macrophages can act as antigenpresenting cells (APCs), collaborating with the early development of acquired immunity (27).

Under homeostatic conditions bone marrow derived monocytes enter tissue and differentiate into tissue specialized macrophages, including Langerhans cells (LCs), in the extracellular matrix of the mucosa $(5,28)$. Resident oral mucosal macrophages exhibit an array of diverse functions depending on different factors they encounter in their environment, including tissue architecture and microbiota $(4,29)$. A small portion of oral Langerhans cells that originate from monocytes are a more specialized subset of tissue resident macrophages $(30,31)$. Even within the same organ, macrophages can occupy different niches and have different specialized functions (28). Interestingly, recent research suggests that during tissue injury caused by myocardial infarction, stroke, and sepsis local proliferation of macrophages dominate over macrophage recruitment (32).

Oral macrophages are important for bridging the innate and adaptive immune response. Macrophages and oral Langerhans cells express high levels of MHCII and CD80/CD86, ingest particulate antigen and can present it to $\mathrm{T}$ cells $(29,31,33,34)$. Interestingly, oral LCs, unlike other tissue macrophages and similarly to dendritic cells, can migrate to lymph nodes to present antigen to $\mathrm{T}$ cells (30). Oral macrophages are also able to prime systemic immunity, as has been shown through systemic protection after sublingual vaccine administration and systemic antibody response to periodontal pathogens (4). In addition, when macrophages are depleted and mice infected intraorally with the periodontal pathogen Porphomonas gingivalis, specific antibody and cytokine responses are decreased, indicating the importance of macrophages in the adaptive immune response to oral bacteria (12).

\section{MACROPHAGE POLARIZATION IN THE ORAL ENVIRONMENT}

Macrophages can polarize to a variety of phenotypes ranging from alternatively polarized M2 macrophages to classically polarized M1 macrophagesin vitro, which is well described in the literature $(6,28,35,36)$. In general, the M1 phenotype promotes a Th1 response and vigorous microbicidal and tumoricidal activity. In contrast, an M2 phenotype helps parasite clearance, dampens inflammation, promotes tissue remodeling, tumor progression, and possesses immuneregulatory functions (37). In reality, in vivo macrophages do not exist on dipoles as a population, but in a continuation of activation. The macrophage phenotype is plastic and in disease and in health there can be wide variety of multiple phenotypes present spanning from strict M1 to strict M2 and anywhere in between (38-40). Throughout this review in vivo macrophages will be referred to as M1-like or M2-like for this reason.

In the oral cavity, as with elsewhere in the body, predominate M1-like activation is generally associated with inflammatory diseases and predominate M2-like activation is associated with cancer (Figure 1) $(6,36)$. Indeed, dysregulation of the M1/M2 balance can lead to the progression of the inflammatory response and malignant oral diseases such as oral lichen planus and oral squamous cell carcinoma (SCC). M1-like macrophages can aid the progression of oral lichen planus and potentially induce malignant transformation. Conversely, M2-like macrophages [often termed tumor associated macrophages (TAMs)] aid SCC progression and favors an immunosuppressive tumor microenvironment (41). This is a general and well-known phenomenon of macrophages in many tumors, not just those in the oral cavity, with recent thorough reviews and so won't be 


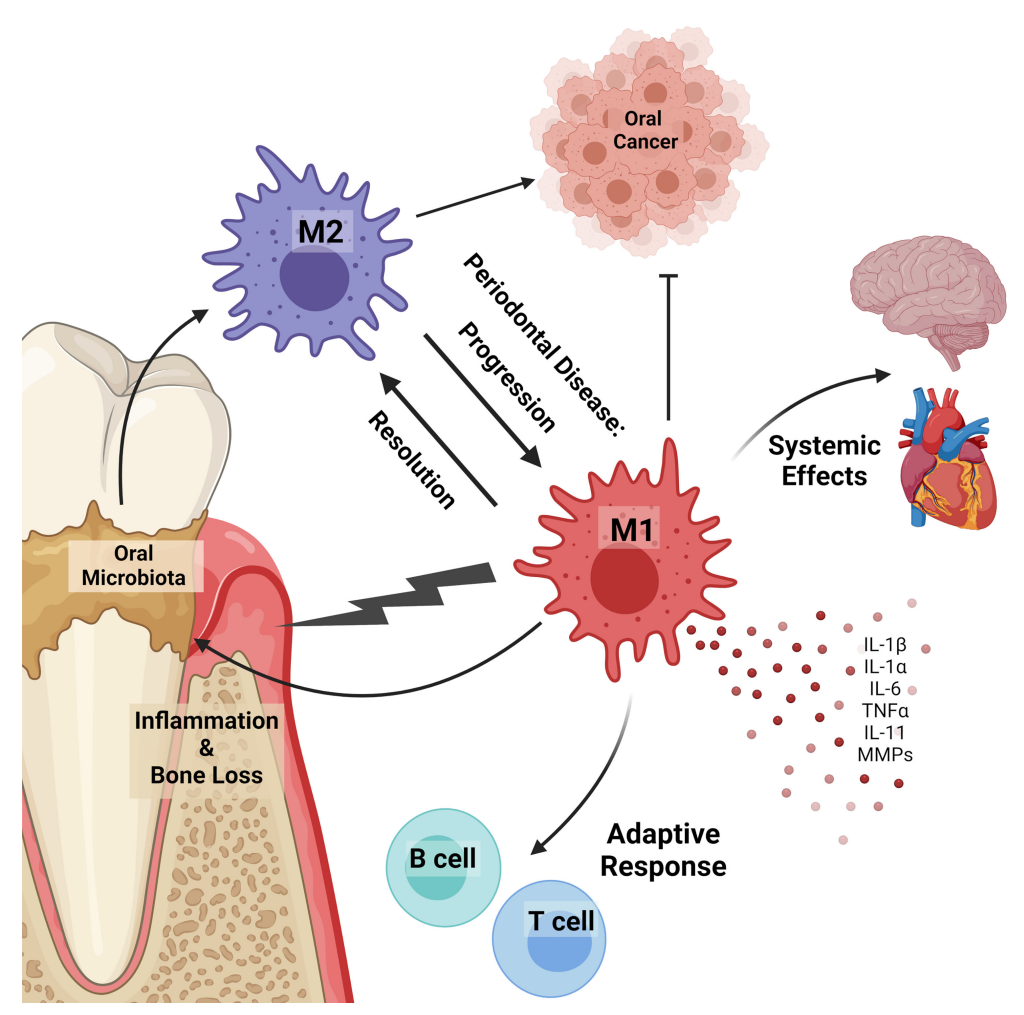

FIGURE 1 | Macrophages in the oral cavity. Macrophages can polarize in response to oral microbiota and in disease. Inflammatory (M1) macrophages promote inflammation, alveolar bone loss, disease progression, microbiota dysbiosis, and prevent tumor development. Alternatively activated (M2) macrophages contribute to cancer progression and resolution of inflammatory diseases. Macrophages in the oral cavity are linked to systemic diseases, including those in the heart and brain. Inflammatory macrophages release pro-inflammatory cytokines (IL-1 $\beta, I L-1 \alpha$, IL-6, TNF $\alpha, I L-11)$ and matrix metalloproteases (MMPs) that contribute to inflammation and alveolar bone loss. Under homeostatic and disease conditions oral macrophages work to bridge the innate and adaptive immune response by expressing antigens to $\mathrm{B}$ and $\mathrm{T}$ lymphocytes.

discussed further here $(40,42)$. Macrophage polarization can be driven by different bacterial species, microbial components, and host immune mediators $(38,43)$. For example, in murine macrophages a differential M1 or M2 profile occurs in response to representative Gram-negative or Gram-positive gastrointestinal bacteria, including probiotic strains, pathogens, commensals, and strains of food origin (44). In the oral environment, endotoxin and other bacterial products present in sterilized saliva polarize macrophages to an M1-like phenotype, with increased production of pro-inflammatory cytokines (45). Specific oral strains can also elicit unique responses, with data showing oral commensals generally elicit an M2-like phenotype while oral pathogens elicit a more M1-like phenotype (46). Furthermore, a switch from an predominantly M2-like phenotype to a predominantly M1-like phenotype is a potential mechanism for the advancement of periodontal disease (42). Studies using germ-free and specific pathogen free mice have also shed light on the ability of the oral microbiota to differentially modify phagocytes and their responses in vivo. For example, there is a significant decrease in IL-1 $\beta$, an inflammatory cytokine mainly produced by macrophages, in germ free (GF) mice compare to specific pathogen free (SPF) mice (47), and oral Langerhans cells are significantly reduced in GF mice compared to SPF mice, but after microbial recolonization their numbers were restored (34).

\section{MACROPHAGES IN PERIODONTAL DISEASE}

Periodontal disease is a progressive inflammatory disease that results from dysbiosis in the microbiota and an overreactive immune response $(48,49)$. It is a complex disease, resulting from a myriad of different factors including genetics, environment, and microbes, with the microbial load from health to disease increasing from $10^{2}$ bacteria in health to $10^{8}$ bacteria in periodontitis (47). The importance of macrophages in the development and progression of periodontal disease has been shown through depletion of macrophages in a mouse model of periodontal disease, resulting in reduced $P$. gingivalis induced bone resorption (12). Although depletion of macrophages prevents bone resorption, it can also impairs bone regeneration (50). Data suggests aberrant expression of macrophage genes may affect their activation state and expression of signaling molecules, thereby contributing to disease progression (51). 
Indeed, many factors released by macrophages can be involved in periodontitis-associated alveolar bone loss. For example, inflammatory cytokines produced in high levels by macrophages including IL-1 $\beta$, IL- $1 \alpha$, TNF $\alpha$ and IL-6 can activate osteoclasts making it likely that they play an important role in disease-induced bone resorption (26), and IL-1 $\beta$ is known to increase in human groups in association with an increased ratio of inflammatory macrophages over alternatively activated macrophages (52). In addition to cytokines, macrophages release a number of matrix metalloproteinases that are involved in degradation of the extracellular matrix and are important proteases involved in the progression of periodontal disease (53). Macrophage cytokines TNF $\alpha$, IL-1, and IL-6 stimulate MMPs, all of which are expressed at higher levels in diseased periodontal tissue (54-56), with again some MMPs being linked to an increased M1/M2 ratio during disease (52). Together this shows the important role that macrophages and the myriad of factors they express can play in the destructive properties of periodontal disease.

As described above, macrophages can polarize to a broad range of phenotypes. On one end of this spectrum lays M1 macrophages, which generally have more inflammatory and microbicidal characteristics. Macrophages can polarize to an M1 phenotype through stimulation with IFN $\gamma$ and TLR ligand interaction, as well as in response to periodontopathic bacteria. Macrophages stimulated with $P$. gingivalis or $P$. gingivalis LPS generally polarize to an M1-like inflammatory phenotype as shown by increased levels of pro-inflammatory cytokines and M1 specific surface markers $(46,57,58)$. Mice infected with $P$. gingivalis also show increased levels of M1-like macrophages compared to M2like macrophages (12). Aggregetibacter actinomycetecometans, another pathogen highly associated with periodontal disease, has also been shown to polarize macrophages to an M1-like phenotype $(46,59)$.

Periodontal disease is an inflammatory disease, so logically inflammatory macrophages (i.e., M1 macrophages) would infiltrate the periodontal tissue during disease: in recent years many studies have shown that this is indeed the case. A human experimental gingivitis study from Topoll and co-workers in 1989 was one of the first to show a decrease in anti-inflammatory macrophages and an increase in inflammatory macrophages (60). Now there have been multiple human studies showing increases of inflammatory M1-like macrophages in periodontal disease in comparison to healthy controls $(42,52,61)$. These M1-like macrophages contribute to the inflammatory environment, promoting dysbiosis of the microbial community and periodontal disease progression (10). Macrophage interactions with normally commensal oral bacteria can also change, as seen with the increased, rather than decreased, survival of Streptococcus gordonii within IFN $\gamma / \mathrm{LPS}$ stimulated macrophages (62). Animal studies have also found that if the inflammatory response, especially by macrophages, is treated then progression of periodontal disease can be inhibited. One promising treatment is with the anti-inflammatory agent Resolvin-E1, which can resolve inflammation and regenerate bone and soft tissue to a healthy state (63). Such studies with anti-inflammatories have further illuminated periodontal disease as an inflammatory disease. Importantly, if alternatively activated, M2, macrophages are stimulated in vivo or injected into animal models of periodontal disease they promote healing and dampen osteoclast activity, thereby reducing bone resorption $(50,64,65)$. On the other hand, $P$. gingivalis may promote the activation of macrophages into M2-like TAMs when combined with an OSCC microenvironment that can induce and promote OSCC growth (66).

\section{SYSTEMIC EFFECTS OF ORAL MACROPHAGES}

There has been a recent increase in research focusing on the systemic implications of periodontal disease $(48,67-70)$. It is known that oral microbes can be found in extraoral locations after gaining access to the circulation, and periodontal diseaseassociated microbes have been found in multiple extra-oral sites including atherosclerotic plaques $(71,72)$ and the brain, showing they also can cross the blood-brain barrier $(73,74)$. Moreover, $P$. gingivalis appears capable of invading and converting myeloid-derived dendritic cells (mDCs) to an atherogenic phenotype in humans with chronic periodontitis (75). Along this line, the uptake of low-density lipoprotein (LDL) by transmigrated macrophages is enhanced in the presence of bacteria leading to accelerated foam cell formation and atherogenesis (48).

In addition to extra-oral bacteria affecting macrophages and systemic disease, the elevated systemic inflammation associated with periodontitis may have multiple systemic complications. For example, periodontal bacteria increase systemic IL-6 levels, driving the expansion of osteoclast precursors (OCPs) which can traffic to sites of bone resorption and differentiate into mature osteoclasts (76), suggesting that changes in the bone marrow may link periodontitis to other bone loss disorders, such as rheumatoid arthritis (77). Additionally, as individuals age there is increased inflammation in a nominally resting state and linked to this there is an increase in primarily M1 macrophage activation with age in nonhuman primates (78). Aging can enrich the gingival environment in anaerobic species leading to a dysregulated and persistent immunoinflammatory response (79). In this context, increases in prevalence and severity of periodontal disease have long been associated with aging (79). Recent evidence finds this long-associated age-related increase in periodontal disease is dependent, at least in part, on these agerelated changes in macrophage activation towards an M1-like phenotype (11).

\section{NEUTROPHIL PHYSIOLOGY}

Neutrophils are the most abundant circulating leukocyte, which are among the first cells to respond to bacterial infections and pro-inflammatory signals. Neutrophils are produced in the bone marrow with 1 to $2 \times 10^{11}$ neutrophils normally generated per day 
in an adult human (80). Mature neutrophils can be found in the bone marrow as the bone marrow reserve, circulating through the blood, or in tissues as resident neutrophils (81).

After production, mature neutrophils will remain in the bone marrow for about 5 days, forming the bone marrow reserve from which neutrophils can be rapidly mobilized in case of infection $(82,83)$. Once neutrophils cross the bone marrow sinusoidal endothelium (about $10^{9}$ neutrophils/kg body weight exit the bone marrow daily in humans) they enter the sinusoids and eventually migrate out into the general circulation $(84,85)$. Some of these neutrophils migrate into tissues, including through mucosal membranes and areas requiring constant immune surveillance, such as the oral cavity.

In human blood, neutrophils are generally believed to have a short half-life of about 8 hours, according to experiments in which neutrophils were labelled ex vivo and then evaluated in vivo (86). However, a study that labeled cells in vivo via deuterium-labelled water reported a surprising lifespan for circulatory neutrophils in humans of up to 5.4 days (87). Yet this paper's methods received some criticism as orally administrated deuterium-labelled water would likely label bone marrow neutrophils as well as circulating neutrophils, therefore skewing their supposed longevity in blood (88). Another group proposes that the slow kinetics of labeled cells reported in Pillay 2010 may be due to the slow production of neutrophils in the bone marrow, rather than a long half-life in the blood $(18,89,90)$.

These dynamic cells perform a wide range of protective functions, including chemotaxis toward stimuli, extravasation from vasculature and antimicrobial actions through phagocytosis, granule release, reactive oxygen species (ROS) production, and NETosis (91, 92). While neutrophils have traditionally been thought of as stable differentiated cells, evidence now demonstrates they are dynamic cells able to change their characteristics and behavior throughout their lifespan or in differing environments. Their potential responses can vary widely and change according to local signals released during acute or chronic inflammatory conditions, injury, infection, cancer, and autoimmunity. This plasticity has led to increasing interest in understanding their functional phenotypic heterogeneity, similar to other immune cell lineages (93).

\section{NEUTROPHILS IN THE ORAL ENVIRONMENT}

In the oral cavity, neutrophils have been identified in periodontal tissues, gingival crevicular fluid, and within dental biofilms (9496). Neutrophils transmigrate through oral mucosa and comprise the majority of innate immune cells recruited to the gingival crevice, composing greater than $90 \%$ of the crevicular cells (97-101). Phenotypic differences between oral neutrophils in health and with location-specific differences are becoming better understood. Compared with circulating neutrophils, oral neutrophils present site-specific gene expression profiles in healthy individuals (102). Human studies have found that generally, oral tissue-resident neutrophils are in a later stage of their life cycle when compared to circulatory neutrophils and present a higher state of activation when compared to circulatory neutrophils, showing higher expression of activation markers CD11b, CD63 and CD66b, as well as higher constitutive ROS levels (103). In periodontal health a spectrum of neutrophil populations have been reported in humans, as characterized by two distinct subtypes of oral neutrophils; a 'parainflammatory 1' population which is similar to naïve blood neutrophils and a 'parainflammatory 2' population which is the more activated phenotype, possibly being in a primed state and may be crucial for response with the symbiotic biofilm of health (22). Another subset of oral neutrophils has also been identified that present with a significant increase in T cell receptor (TCR) expression compared with circulating neutrophils, and these cells show markedly increased recruitment to sites of inflammation. While the exact role of TCR expression in oral neutrophils is unknown, this supports a role for oral neutrophils in crosstalk between the innate and adaptive immune system in the oral cavity (Figure 2) (102).

\section{NEUTROPHILS IN ORAL CANCER}

Myeloid cells can promote tumor progression directly via immune suppression or indirectly via production of angiogenic factors, matrix-degrading enzymes, or growth factors. The most well characterized of these cells are TAMs that have properties of alternatively activated macrophages, or M2-like macrophages as described above (104). In recent years there has been increasing evidence showing phenotypic and functional plasticity in neutrophils, particularly in oral cancers (105). Like TAMs, these tumor-associated neutrophils (TANs) have differential states of activation/differentiation (104) TANs can polarize to antitumorigenic (N1) or pro-tumorigenic (N2) phenotypes. It is important to note that in vivo neutrophils, like macrophages, exist on a spectrum of activation and so in reality ' $\mathrm{N} 1$ ' and 'N2' are better understood as ends of a spectrum rather than discrete categories. In vivo animal studies show this change is largely directed by TGF- $\beta$, which skews differentiation toward the $\mathrm{N} 2$ phenotype. In vitro studies on human blood and tumor samples have found that N1 TANs express higher levels of immune activating cytokines and chemokines, lower levels of arginase, and have a heightened ability to kill tumor cells in vitro. N1 TANs express higher level of CCL3, ICAM-1, and TRAIL. N2 TANs support tumor growth by producing angiogenic factors and matrix-degrading enzymes and suppress the antitumor immune response. They are characterized by increased expression of gene markers, such as MMP9, VEGF-A, and BV8, as well as the decreased expression of CCL3, ICAM-1, and TRAIL $(106,107)$. Knowledge of TAN and circulating neutrophils in the context of head and neck cancer (HNC) of the oral cavity is somewhat limited, and even less is known about the oral neutrophils which populate the environment closely associated with evolving HNC. These oral neutrophils represent a unique population with functional and phenotypic proprieties distinct from other compartments such as the tumor or mucosal tissue (108). Localization of the oral neutrophils and neutrophil-derived products along with the tumor-derived microenvironment rich is 


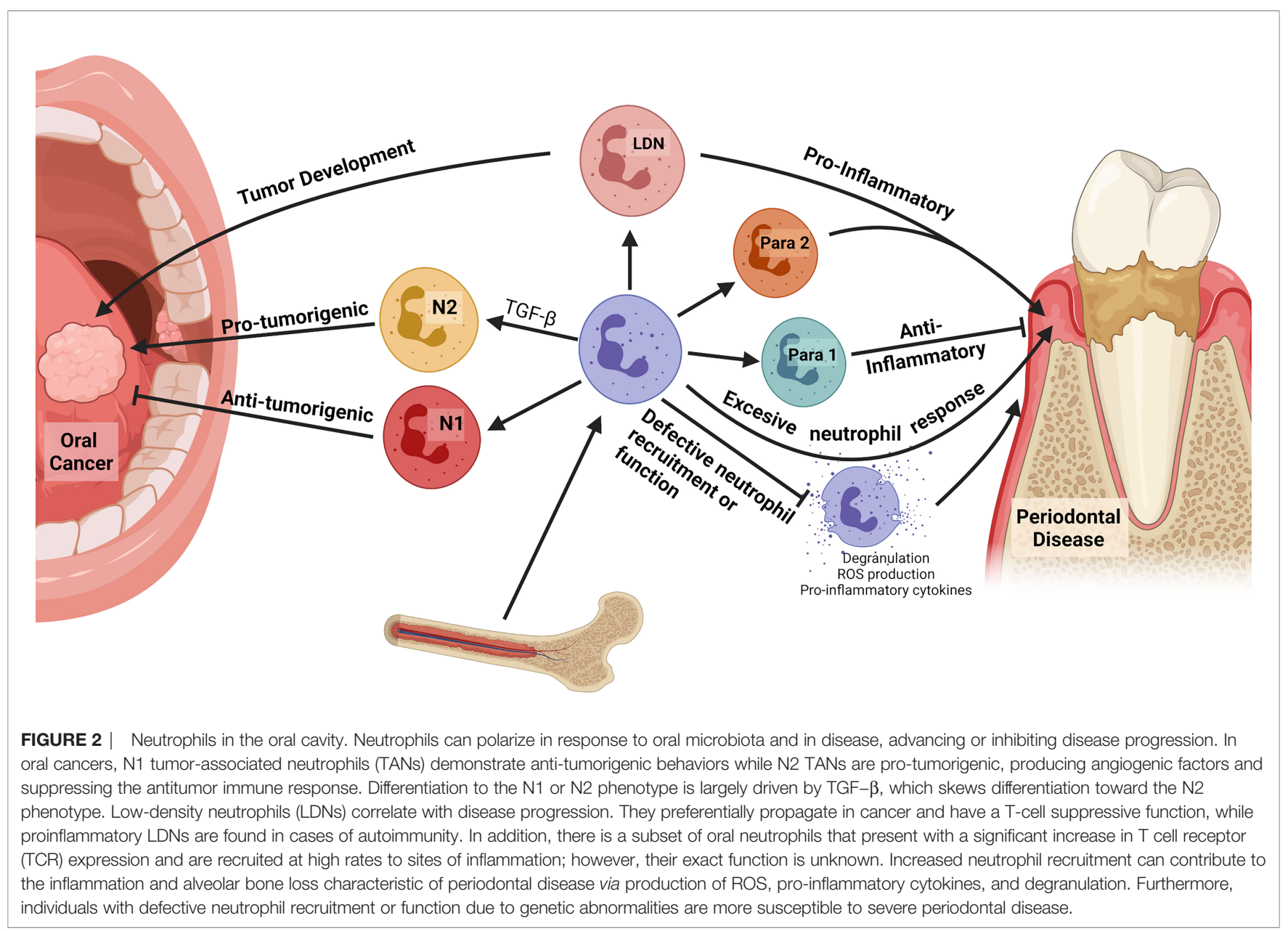

growth factors and cytokines may influence tumor development and differential neutrophil plasticity. For example, oral neutrophils from patients with untreated oral cancer demonstrate distinct functional properties with lower chemotactic ability, superoxide production and reduced killing of microbes (109). Understanding phenotypic differences and properties of oral neutrophils in oral cancer provides a relevant measure of local response in the tumor environment with high prognostic value.

Neutrophil granule changes have also been demonstrated to reflect differential phenotypes as during some inflammatory states or other disease conditions, there exists a subset population of neutrophils which co-sediment with peripheral blood mononuclear cells (PBMCs) due to their unique granularity compared to normal mature neutrophils known as low-density neutrophils (LDNs) (110). LDN numbers correlate with pathological conditions such as rheumatoid arthritis, lupus and cancers including potentially in the early phase of oral squamous cell carcinoma development (111113). Their overall function is dependent on inflammatory stimuli. For example, a proinflammatory LDN phenotype has been described in vivo animal studies for LDNs in autoimmunity and infection while in cancer LDNs primarily have a T cell suppressive function and are often referred to as myeloid-derived suppressor cells $(111,114)$.

\section{NEUTROPHILS IN PERIODONTAL DISEASE AND INFLAMMATION}

The association between periodontal disease and neutrophil presence is well established. The human oral cavity has a constant bacterial presence that is kept under control by equally constant immune surveillance. Neutrophils comprise the majority of innate immune cells recruited to the gingival crevice and tissue to maintain physiological health, while documented increases in the number of oral neutrophils during periodontal disease which correlates with clinical severity in response to the dysbiotic biofilm and inflammatory changes $(98,101,102,115)$. A lack of neutrophil infiltration into the oral cavity or defects of neutrophil function lead to an increase in periodontal disease (9). Congenital defects in neutrophil development or egress from the bone marrow resulting in significant neutropenia are linked to severe periodontal disease (116). Likewise, defective extravasation of neutrophils into tissue and impaired immune response are also linked to increased periodontal disease. For example, Leukocyte adhesion deficiency are a group of congenital disorders in which neutrophils have defective expression or function of adhesion molecules involved in attachment and migration through the vascular endothelium, resulting in neutrophilia with few neutrophils in peripheral 
tissues leading to subsequent recurrent infections including severe periodontal disease (117). On the other hand, increased neutrophil recruitment in attempt to control the dysbiotic biofilm characteristic of periodontal disease or dysregulation of appropriate trafficking and resolution of the neutrophil response contributes to much of the tissue damage in periodontal disease (10). Many of the oral microbes abundant in the dysbiotic community of periodontal disease are able to disarm or impair local neutrophil responses, also rendering these cells ineffective in the gingival tissues $(118,119)$.

A variety of studies have suggested that neutrophils during periodontal disease are heterogenous populations which likely respond to local microenvironment and microbe cues. Classical studies have revealed that circulating neutrophils from patients with periodontal disease have impaired chemotactic ability, responsiveness and directionality along with changes in surface expression of CD11b or CD16 (120-122), which suggest potential of functional neutrophil subsets. Recent detailed comparative analysis of oral neutrophil surface markers has further revealed distinct upregulation of specific markers during periodontitis characterized by a pro-inflammatory signature along with a functionally activated phenotype with elevated degranulation, phagocytosis, ROS production and NET formation (22, 103, 123). Studies on blood and oral rinses from healthy and chronic periodontitis patients have revealed differential expression of neutrophil surface markers in different biological compartments (124) and during transmigration (125), however ongoing studies are required to identify definitively if observed molecular changes and phenotypic features in oral neutrophils during disease represent active subset switching in response to stimuli or a true differentiated population.

Despite appropriate clinical therapy, a subset of periodontitis patients do not respond effectively and present with continuing disease progression and clinical attachment loss that does not correlate with plaque levels, microbiology assessments, and treatment compliance. Such patients are diagnosed with refractory periodontitis (RP) (126). A unique hyperactive oral neutrophil phenotype characterized by increased potential for ROS production has been identified in these RP patients. These cells were found to produce ROS at a level approaching the maximal capability of the cells (127). While appropriate ROS production is crucial for effective bacterial killing, excessive ROS can contribute to periodontal attachment loss by damaging the extracellular connective tissue (128).

While characteristic neutrophil surface marker signatures have been demonstrated, a distinct neutrophil-specific marker has been reported to be associated with periodontal disease. CD177, also called neutrophil antigen B1 (NB1) or human neutrophil antigen 2a (HNA-2a), is a glycosylphosphatidylinositol (GPI)-linked glycoprotein expressed on the plasma membrane and in granule membranes of neutrophils (129). A high proportion of CD177expressing neutrophils have also been found in the gingival crevicular fluid (GCF) of periodontitis patients. The proportion of CD177-expressing neutrophils in circulation varies between individuals with a relatively stable bimodal, or occasionally trimodal, expression pattern $(130,131)$. In humans, the proportion of CD177-expressing neutrophils ranges from $0 \%$ and
$100 \%$ of circulating neutrophils. CD177 can interact with PECAM1 (expressed on endothelial cells, platelets, monocytes, and granulocytes), which is a key player in neutrophil migration from bloodstream to tissue (132). The proportions of CD $177^{+}$ neutrophils is higher in GCF from periodontitis patients, as compared to blood from the same donor and this accumulation of CD $177^{+}$neutrophils in inflammatory exudate was not seen in two different models of aseptic inflammation, suggesting that this is a periodontitis specific phenotype. Furthermore, the CD $177^{+}$ neutrophil subtype does not accumulate in the GCF of healthy subjects (133).

As periodontal disease is considered an inflammatory condition with systemic associations, neutrophil phenotypes related to inflammation are likely relevant to health in the oral cavity as well as systemically at distant sites. Recent work has demonstrated that oral inflammation occurring during human experimental gingivitis or ligature induced models of murine periodontitis has systemic effects to produce an exacerbated immune response at a secondary site during a secondary insult (134). This supports a larger role of oral inflammation and particularly neutrophil-derived changes broadly throughout the body.

\section{SUMMARY}

There is an abundance of work highlighting the importance of phagocytic cells, including macrophages and neutrophils, under conditions of health and disease in the oral environment. Both of these cell types play specialized roles in part by polarizing to a variety of phenotypes to alter their phenotypic responses in health and disease. As described in this review, a key role for such polarization in the context of oral diseases, including periodontal disease and oral cancer is well documented. However, while there is increased understanding of the roles for innate immune cell polarization in the oral environment in recent years, their remains a lack of insight into molecular mechanisms fueling the responses of these phagocytes. Future characterization of the plasticity of innate immune cells will provide important information to decipher their detailed roles in driving pathogenic conditions in the oral cavity.

\section{AUTHOR CONTRIBUTIONS}

SM, NA, AE, MV, and JK all contributed to the writing and editing of the manuscript. All authors contributed to the article and approved the submitted version.

\section{FUNDING}

The authors received funding from the following, NIH grants F31DE029400 (SM), F31DE030705 (NA), R01DE027073 (MV), R01DE028307 (JK) and T32DE023526, as well as Fondo Nacional de Ciencia y Tecnología FONDECYT 1180666 (AE).

\section{ACKNOWLEDGMENTS}

Figures were created with BioRender.com. 


\section{REFERENCES}

1. Verma D, Garg PK, Dubey AK. Insights Into the Human Oral Microbiome. Arch Microbiol (2018) 200:525-40. doi: 10.1007/s00203-018-1505-3

2. Belkaid Y, Harrison OJ. Homeostatic Immunity and the Microbiota. Immunity (2017) 46:562-76. doi: 10.1016/j.immuni.2017.04.008

3. Hooper LV, Littman DR, Macpherson AJ. Interactions Between the Microbiota and the Immune System. Science (2012) 336:1268-73. doi: $10.1126 /$ science. 1223490

4. Moutsopoulos NM, Konkel JE. Tissue-Specific Immunity at the Oral Mucosal Barrier. Trends Immunol (2018) 39:276-87. doi: 10.1016/ j.it.2017.08.005

5. Culter CW, Jotwani R. Dendritic Cells at the Oral Mucosal Interface. J Dent Res (2006) 85:678-89. doi: 10.1177/154405910608500801

6. Merry R, Belfield L, Mcardle P, Mclennan A, Crean S, Foey A. Oral Health and Pathology: A Macrophage Account. Br J Oral Maxillofac Surg (2012) 50:2-7. doi: 10.1016/j.bjoms.2010.10.020

7. Sanz M, Beighton D, Curtis MA, Cury JA, Dige I, Dommisch H, et al. Role of Microbial Biofilms in the Maintenance of Oral Health and in the Development of Dental Caries and Periodontal Diseases. Consensus Report of Group 1 of the Joint EFP/ORCA Workshop on the Boundaries Between Caries and Periodontal Disease. J Clin Periodontol (2017) 44 Suppl 18:S5-S11. doi: 10.1111/jcpe.12682

8. Meyle J, Dommisch H, Groeger S, Giacaman RA, Costalonga M, Herzberg M. The Innate Host Response in Caries and Periodontitis. J Clin Periodontol (2017) 44:1215-25. doi: 10.1111/jcpe.12781

9. Moutsopoulos NM, Konkel J, Sarmadi M, Eskan MA, Wild T, Dutzan N, et al. Defective Neutrophil Recruitment in Leukocyte Adhesion Deficiency Type I Disease Causes Local IL-17-Driven Inflammatory Bone Loss. Sci Transl Med (2014) 6:229ra240. doi: 10.1126/scitranslmed.3007696

10. Lamont RJ, Koo H, Hajishengallis G. The Oral Microbiota: Dynamic Communities and Host Interactions. Nat Rev Microbiol (2018) 16:745-59. doi: 10.1038/s41579-018-0089-x

11. Clark D, Halpern B, Miclau T, Nakamura M, Kapila Y, Marcucio R. The Contribution of Macrophages in Old Mice to Periodontal Disease. J Dent Res (2021) 100:220345211009463. doi: 10.1177/00220345211009463

12. Lam RS, O'brien-Simpson NM, Lenzo JC, Holden JA, Brammar GC, Walsh KA, et al. Macrophage Depletion Abates Porphyromonas GingivalisInduced Alveolar Bone Resorption in Mice. J Immunol (2014) 193:234962. doi: 10.4049/jimmunol.1400853

13. Wynn TA, Chawla A, Pollard JW. Macrophage Biology in Development, Homeostasis and Disease. Nature (2013) 496:445-55. doi: 10.1038/nature12034

14. Murray PJ, Allen JE, Biswas SK, Fisher EA, Gilroy DW, Goerdt S, et al. Macrophage Activation and Polarization: Nomenclature and Experimental Guidelines. Immunity (2014) 41:14-20. doi: 10.1016/j.immuni.2014.06.008

15. Gordon S, Pluddemann A. Tissue Macrophages: Heterogeneity and Functions. BMC Biol (2017) 15:53. doi: 10.1186/s12915-017-0392-4

16. Tsuda Y, Takahashi H, Kobayashi M, Hanafusa T, Herndon DN, Suzuki F. Three Different Neutrophil Subsets Exhibited in Mice With Different Susceptibilities to Infection by Methicillin-Resistant Staphylococcus Aureus. Immunity (2004) 21:215-26. doi: 10.1016/j.immuni.2004.07.006

17. Fridlender ZG, Buchlis G, Kapoor V, Cheng G, Sun J, Singhal S, et al. CCL2 Blockade Augments Cancer Immunotherapy. Cancer Res (2010) 70:109-18. doi: 10.1158/0008-5472.CAN-09-2326

18. Pillay J, Ramakers BP, Kamp VM, Loi AL, Lam SW, Hietbrink F, et al. Functional Heterogeneity and Differential Priming of Circulating Neutrophils in Human Experimental Endotoxemia. J Leukoc Biol (2010) 88:211-20. doi: 10.1189/jlb.1209793

19. Puga I, Cols M, Barra CM, He B, Cassis L, Gentile M, et al. B Cell-Helper Neutrophils Stimulate the Diversification and Production of Immunoglobulin in the Marginal Zone of the Spleen. Nat Immunol (2011) 13:170-80. doi: 10.1038/ni.2194

20. Matthews JB, Wright HJ, Roberts A, Ling-Mountford N, Cooper PR, Chapple IL. Neutrophil Hyper-Responsiveness in Periodontitis. J Dent Res (2007) 86:718-22. doi: 10.1177/154405910708600806

21. Ling MR, Chapple IL, Matthews JB. Peripheral Blood Neutrophil Cytokine Hyper-Reactivity in Chronic Periodontitis. Innate Immun (2015) 21:714-25. doi: $10.1177 / 1753425915589387$
22. Fine N, Hassanpour S, Borenstein A, Sima C, Oveisi M, Scholey J, et al. Distinct Oral Neutrophil Subsets Define Health and Periodontal Disease States. J Dent Res (2016) 95:931-8. doi: 10.1177/0022034516645564

23. Fine N, Chadwick JW, Sun C, Parbhakar KK, Khoury N, Barbour A, et al. Periodontal Inflammation Primes the Systemic Innate Immune Response. J Dent Res (2020) 100(3):318-25. doi: 10.1177/0022034520963710

24. Varol C, Mildner A, Jung S. Macrophages: Development and Tissue Specialization. Annu Rev Immunol (2015) 33:643-75. doi: 10.1146/ annurev-immunol-032414-112220

25. Vladimer GI, Marty-Roix R, Ghosh S, Weng D, Lien E. Inflammasomes and Host Defenses Against Bacterial Infections. Curr Opin Microbiol (2013) 16:23-31. doi: 10.1016/j.mib.2012.11.008

26. Metzger Z. Macrophages in Periapical Lesions. Endod Dent Traumatol (2000) 16:1-8. doi: 10.1034/j.1600-9657.2000.016001001.x

27. Von Delwig A, Bailey E, Gibbs DM, Robinson JH. The Route of Bacterial Uptake by Macrophages Influences the Repertoire of Epitopes Presented to CD4 T Cells. Eur J Immunol (2002) 32:3714-9. doi: 10.1002/1521-4141 (200212)32:12<3714::AID-IMMU3714>3.0.CO;2-Y

28. Gordon S, Pluddemann A, Martinez Estrada F. Macrophage Heterogeneity in Tissues: Phenotypic Diversity and Functions. Immunol Rev (2014) 262:36-55. doi: 10.1111/imr.12223

29. Hasturk H, Kantarci A, Van Dyke TE. Oral Inflammatory Diseases and Systemic Inflammation: Role of the Macrophage. Front Immunol (2012) 3:118. doi: 10.3389/fimmu.2012.00118

30. Doebel T, Voisin B, Nagao K. Langerhans Cells - The Macrophage in Dendritic Cell Clothing. Trends Immunol (2017) 38:817-28. doi: 10.1016/ j.it.2017.06.008

31. Hovav AH. Mucosal and Skin Langerhans Cells - Nurture Calls. Trends Immunol (2018) 39:788-800. doi: 10.1016/j.it.2018.08.007

32. Hoyer FF, Naxerova K, Schloss MJ, Hulsmans M, Nair AV, Dutta P, et al. Tissue-Specific Macrophage Responses to Remote Injury Impact the Outcome of Subsequent Local Immune Challenge. Immunity (2019) 51 (5):899-914.e7. doi: 10.1016/j.immuni.2019.10.010

33. Hasseus B, Jontell M, Bergenholtz G, Dahlgren UI. Langerhans Cells From Human Oral Epithelium Are More Effective at Stimulating Allogeneic T Cells In Vitro Than Langerhans Cells From Skin. Clin Exp Immunol (2004) 136:483-9. doi: 10.1111/j.1365-2249.2004.02469.x

34. Capucha T, Koren N, Nassar M, Heyman O, Nir T, Levy M, et al. Sequential BMP7/TGF-Betal Signaling and Microbiota Instruct Mucosal Langerhans Cell Differentiation. J Exp Med (2018) 215:481-500. doi: 10.1084/ jem.20171508

35. Mosser DM, Edwards JP. Exploring the Full Spectrum of Macrophage Activation. Nat Rev Immunol (2008) 8:958-69. doi: 10.1038/nri2448

36. Murray PJ. Macrophage Polarization. Annu Rev Physiol (2017) 79:541-66. doi: 10.1146/annurev-physiol-022516-034339

37. Biswas SK, Mantovani A. Macrophage Plasticity and Interaction With Lymphocyte Subsets: Cancer as a Paradigm. Nat Immunol (2010) 11:88996. doi: $10.1038 /$ ni. 1937

38. Sica A, Mantovani A. Macrophage Plasticity and Polarization: In Vivo Veritas. J Clin Invest (2012) 122:787-95. doi: 10.1172/JCI59643

39. Brundu S, Fraternale A. Polarization and Repolarization of Macrophages. J Clin Cell Immunol (2015) 6(2):1000319. doi: 10.4172/2155-9899.1000319

40. Atri C, Guerfali FZ, Laouini D. Role of Human Macrophage Polarization in Inflammation During Infectious Diseases. Int J Mol Sci (2018) 19(6):1801. doi: $10.3390 / \mathrm{ijms} 19061801$

41. Sica A, Allavena P, Mantovani A. Cancer Related Inflammation: The Macrophage Connection. Cancer Lett (2008) 267:204-15. doi: 10.1016/ j.canlet.2008.03.028

42. Yu T, Zhao L, Huang X, Ma C, Wang Y, Zhang J, et al. Enhanced Activity of the Macrophage M1/M2 Phenotypes and Phenotypic Switch to M1 in Periodontal Infection. J Periodontol (2016) 87:1092-102. doi: 10.1902/ jop.2016.160081

43. Benoit M, Desnues B, Mege JL. Macrophage Polarization in Bacterial Infections. J Immunol (2008) 181:3733-9. doi: 10.4049/jimmunol.181.6.3733

44. Christoffersen TE, Hult LT, Kuczkowska K, Moe KM, Skeie S, Lea T, et al. In Vitro Comparison of the Effects of Probiotic, Commensal and Pathogenic Strains on Macrophage Polarization. Probiot Antimicrob Proteins (2014) 6:1-10. doi: 10.1007/s12602-013-9152-0 
45. Pourgonabadi S, Muller HD, Mendes JR, Gruber R. Saliva Initiates the Formation of Pro-Inflammatory Macrophages In Vitro. Arch Oral Biol (2017) 73:295-301. doi: 10.1016/j.archoralbio.2016.10.012

46. Huang CB, Alimova Y, Ebersole JL. Macrophage Polarization in Response to Oral Commensals and Pathogens. Pathog Dis (2016) 74(3):ftw011. doi: 10.1093/femspd/ftw011

47. Dixon DR, Bainbridge BW, Darveau RP. Modulation of the Innate Immune Response Within the Periodontium. Periodontol 2000 (2004) 35:53-74. doi: 10.1093/femspd/ftw011

48. Hajishengallis G. Periodontitis: From Microbial Immune Subversion to Systemic Inflammation. Nat Rev Immunol (2015) 15:30-44. doi: 10.1038/nri3785

49. Lamont RJ, Hajishengallis G. Polymicrobial Synergy and Dysbiosis in Inflammatory Disease. Trends Mol Med (2015) 21:172-83. doi: 10.1016/ j.molmed.2014.11.004

50. Viniegra A, Goldberg H, Cil C, Fine N, Sheikh Z, Galli M, et al. Resolving Macrophages Counter Osteolysis by Anabolic Actions on Bone Cells. J Dent Res (2018) 97(10):1160-9. doi: 10.1177/0022034518777973

51. Jiang Y, Fu J, Du J, Luo Z, Guo L, Xu J, et al. DNA Methylation Alterations and Their Potential Influence on Macrophage in Periodontitis. Oral Dis (2020) 00:1-15. doi: 10.1111/odi.13654

52. Yang J, Zhu Y, Duan D, Wang P, Xin Y, Bai L, et al. Enhanced Activity of Macrophage M1/M2 Phenotypes in Periodontitis. Arch Oral Biol (2018) 96:234-42. doi: 10.1016/j.archoralbio.2017.03.006

53. Franco C, Patricia HR, Timo S, Claudia B, Marcela H. Matrix Metalloproteinases as Regulators of Periodontal Inflammation. Int $\mathrm{J}$ Mol Sci (2017) 18(2):440. doi: 10.3390/ijms 18020440

54. Stashenko P, Jandinski JJ, Fujiyoshi P, Rynar J, Socransky SS. Tissue Levels of Bone Resorptive Cytokines in Periodontal Disease. J Periodontol (1991) 62:504-9. doi: 10.1902/jop.1991.62.8.504

55. Irwin CR, Myrillas TT. The Role of IL-6 in the Pathogenesis of Periodontal Disease. Oral Dis (1998) 4:43-7. doi: 10.1111/j.1601-0825.1998.tb00255.x

56. Irwin CR, Myrillas TT, Traynor P, Leadbetter N, Cawston TE. The Role of Soluble Interleukin (IL)-6 Receptor in Mediating the Effects of IL-6 on Matrix Metalloproteinase-1 and Tissue Inhibitor of Metalloproteinase-1 Expression by Gingival Fibroblasts. J Periodontol (2002) 73:741-7. doi: 10.1902/jop.2002.73.7.741

57. Shapira L, Champagne C, Van Dyke TE, Amar S. Strain-Dependent Activation of Monocytes and Inflammatory Macrophages by Lipopolysaccharide of Porphyromonas Gingivalis. Infect Immun (1998) 66:2736-42. doi: 10.1128/IAI.66.6.2736-2742.1998

58. Yu S, Ding L, Liang D, Luo L. Porphyromonas Gingivalis Inhibits M2 Activation of Macrophages by Suppressing Alpha-Ketoglutarate Production in Mice. Mol Oral Microbiol (2018) 33:388-95. doi: 10.1111/omi.12241

59. Ando-Suguimoto ES, Benakanakere MR, Mayer MPA, Kinane DF. Distinct Signaling Pathways Between Human Macrophages and Primary Gingival Epithelial Cells by Aggregatibacter Actinomycetemcomitans. Pathogens (2020) 9(4):248. doi: 10.3390/pathogens 9040248

60. Topoll HH, Zwadlo G, Lnage DE, Sorg C. Phenotypic Dynamics of Macrophage Subpopulations During Human Experimental Gingivitis. J Periodontal Res (1989) 24:106-12. doi: 10.1111/j.1600-0765.1989.tb00864.x

61. Higuchi K, Sm Z, Yamashita Y, Ozaki Y, Yoshimura A. Initial Periodontal Treatment Affects Nucleotide-Binding Domain Leucine-Rich RepeatContaining Protein 3 Inflammasome Priming in Peripheral Blood Mononuclear Cells. Arch Oral Biol (2019) 110:104625. doi: 10.1016/j.archoralbio.2019.104625

62. Croft AJ, Metcalfe S, Honma K, Kay JG. Macrophage Polarization Alters Postphagocytosis Survivability of the Commensal Streptococcus Gordonii. Infect Immun (2018) 86(3):e00858-17. doi: 10.1128/IAI.00858-17

63. Hasturk H, Kantarci A, Goguet-Surmenian E, Blackwood A, Andry C, Serhan CN, et al. Resolvin E1 Regulates Inflammation at the Cellular and Tissue Level and Restores Tissue Homeostasis. Vivo J Immunol (2007) 179:7021-9. doi: 10.4049/jimmunol.179.10.7021

64. Zhuang Z, Yoshizawa-Smith S, Glowacki A, Maltos K, Pacheco C, Shehabeldin M, et al. Induction of M2 Macrophages Prevents Bone Loss in Murine Periodontitis Models. J Dent Res (2018) 98(2):200-8. doi: $10.1177 / 0022034518805984$

65. Miao Y, He L, Qi X, Lin X. Injecting Immunosuppressive M2 Macrophages Alleviates the Symptoms of Periodontitis in Mice. Front Mol Biosci (2020) 7:603817. doi: $10.3389 /$ fmolb.2020.603817
66. Liu S, Zhou X, Peng X, Li M, Ren B, Cheng G, et al. Porphyromonas Gingivalis Promotes Immunoevasion of Oral Cancer by Protecting Cancer From Macrophage Attack. J Immunol (2020) 205:282-9. doi: 10.4049/ jimmunol.1901138

67. Debelian GJ, Olsen I, Tronstad L. Systemic Diseases Caused by Oral Microorganisms. Endod Dent Traumatol (1994) 10:57-65. doi: 10.1111/ j.1600-9657.1994.tb00061.x

68. Page RC. The Pathobiology of Periodontal Diseases may Affect Systemic Diseases: Inversion of a Paradigm. Ann Periodontol (1998) 3:108-20. doi: 10.1902/annals.1998.3.1.108

69. Glickman LT, Glickman NW, Moore GE, Goldstein GS, Lewis HB. Evaluation of the Risk of Endocarditis and Other Cardiovascular Events on the Basis of the Severity of Periodontal Disease in Dogs. J Am Vet Med Assoc (2009) 234:486-94. doi: 10.2460/javma.234.4.486

70. Han YW, Wang X. Mobile Microbiome: Oral Bacteria in Extra-Oral Infections and Inflammation. J Dent Res (2013) 92:485-91. doi: 10.1177/ 0022034513487559

71. Haraszthy VI, Zambon JJ, Trevisan M, Zeid M, Genco RJ. Identification of Periodontal Pathogens in Atheromatous Plaques. J Periodontol (2000) 71:1554-60. doi: 10.1902/jop.2000.71.10.1554

72. Szulc M, Kustrzycki W, Janczak D, Michalowska D, Baczynska D, RadwanOczko M. Presence of Periodontopathic Bacteria DNA in Atheromatous Plaques From Coronary and Carotid Arteries. BioMed Res Int (2015) 2015:825397. doi: 10.1155/2015/825397

73. Poole S, Singhrao SK, Kesavalu L, Curtis MA, Crean S. Determining the Presence of Periodontopathic Virulence Factors in Short-Term Postmortem Alzheimer's Disease Brain Tissue. J Alzheimers Dis (2013) 36:665-77. doi: 10.3233/JAD-121918

74. Dominy SS, Lynch C, Ermini F, Benedyk M, Marczyk A, Konradi A, et al. Porphyromonas Gingivalis in Alzheimer's Disease Brains: Evidence for Disease Causation and Treatment With Small-Molecule Inhibitors. Sci $A d v$ (2019) 5:eaau3333. doi: 10.1126/sciadv.aau3333

75. Carrion J, Scisci E, Miles B, Sabino GJ, Zeituni AE, Gu Y, et al. Microbial Carriage State of Peripheral Blood Dendritic Cells (DCs) in Chronic Periodontitis Influences DC Differentiation, Atherogenic Potential. J Immunol (2012) 189:3178-87. doi: 10.4049/jimmunol.1201053

76. Zhao Y, Li Z, Su L, Ballesteros-Tato A, Katz J, Michalek SM, et al. Characterization and Regulation of Osteoclast Precursors Following Chronic Porphyromonas Gingivalis Infection. J Leukoc Biol (2020) 108:1037-50. doi: 10.1002/JLB.1HI0620-230R

77. Hajishengallis G, Chavakis T. Local and Systemic Mechanisms Linking Periodontal Disease and Inflammatory Comorbidities. Nat Rev Immunol (2021) 21:426-40. doi: 10.1038/s41577-020-00488-6

78. Gonzalez OA, Novak MJ, Kirakodu S, Stromberg A, Nagarajan R, Huang CB, et al. Differential Gene Expression Profiles Reflecting Macrophage Polarization in Aging and Periodontitis Gingival Tissues. Immunol Invest (2015) 44:643-64. doi: 10.3109/08820139.2015.1070269

79. Ebersole JL, Graves CL, Gonzalez OA, Dawson D3rd, Morford LA, Huja PE, et al. Aging, Inflammation, Immunity and Periodontal Disease. Periodontol 2000 (2016) 72:54-75. doi: 10.1111/prd.12135

80. Borregaard N. Neutrophils, From Marrow to Microbes. Immunity (2010) 33 (5):657-70. doi: 10.1016/j.immuni.2010.11.011

81. Furze RC, Rankin SM. Neutrophil Mobilization and Clearance in the Bone Marrow. Immunology (2008) 125(3):281-8. doi: 10.1111/j.13652567.2008.02950.x

82. Dancey JT, Deubelbeiss KA, Harker LA, Finch CA. Neutrophil Kinetics in Man. J Clin Invest (1976) 58(3):705-15. doi: 10.1172/JCI108517

83. Sato Y, Van Eeden SF, English D, Hogg JC. Pulmonary Sequestration of Polymorphonuclear Leukocytes Released From Bone Marrow in Bacteremic Infection. Am J Physiol - Lung Cell Mol Physiol (1998) 275(2):L255-61. doi: 10.1152/ajplung.1998.275.2.L255

84. Weiss L. Transmural Cellular Passage in Vascular Sinuses of Rat Bone Marrow. Blood (1970) 36(2):189-208. doi: 10.1182/blood.V36.2.189.189

85. Rankin SM. The Bone Marrow: A Site of Neutrophil Clearance. J Leukocyte Biol (2010) 88(2):241-51. doi: 10.1189/jlb.0210112

86. Galli SJ, Borregaard N, Wynn TA. Phenotypic and Functional Plasticity of Cells of Innate Immunity: Macrophages, Mast Cells and Neutrophils. Nat Immunol (2011) 12:1035-44. doi: 10.1038/ni.2109 
87. Pillay J, Den Braber I, Vrisekoop N, Kwast LM, De Boer RJ, Borghans JA, et al. In Vivo Labeling With 2H2O Reveals a Human Neutrophil Lifespan of 5.4 Days. Blood (2010) 116:625-7. doi: 10.1182/blood-2010-01-259028

88. Tofts PS, Chevassut T, Cutajar M, Dowell NG, Peters AM. Doubts Concerning the Recently Reported Human Neutrophil Lifespan of 5.4 Days. Blood (2011) 117:6050-2. doi: 10.1182/blood-2010-10-310532

89. Li KW, Turner SM, Emson CL, Hellerstein MK, Dale DC. Deuterium and Neutrophil Kinetics. Blood (2011) 117:6052-3. doi: 10.1182/blood-2010-12-322271

90. Mauer AM, Athens JW, Ashenbrucker H, Cartwright GE, Wintrobe MM. Leukokinetic Studies. Ii. A Method For Labeling Granulocytes In Vitro With Radioactive Diisopropylfluorophosphate (Dfp32)*. J Clin Invest (1960) 39 (9):1481-6. doi: 10.1172/JCI104167

91. Lee SH, Dominguez R. Regulation of Actin Cytoskeleton Dynamics in Cells. Mol Cells (2010) 29(4):311-25. doi: 10.1007/s10059-010-0053-8

92. Roberts RE, Hallett MB. Neutrophil Cell Shape Change: Mechanism and Signalling During Cell Spreading and Phagocytosis. In J Mol Sci (2019) 20 (6):1383. doi: 10.3390/ijms20061383

93. Deniset JF, Kubes P. Neutrophil Heterogeneity: Bona Fide Subsets or Polarization States? J Leukoc Biol (2018) 103(5):829-38. doi: 10.1002/ JLB.3RI0917-361R

94. Suzuki T, Sugitam N, Yoshie H, Hara K. Presence of Activated Eosinophils, High IgE and Scd23 Titers in Gingival Crevicular Fluid of Patients With Adult Periodontitis. J Periodontal Res (1995) 30(3):159-66. doi: 10.1111/ j.1600-0765.1995.tb01268.x

95. Hirschfeld J, Dommisch H, Skora P, Horvath G, Latz E, Hoerauf A, et al. Neutrophil Extracellular Trap Formation in Supragingival Biofilms. Int $J$ Med Microbiol (2015) 305(4-5):453-63. doi: 10.1016/j.ijmm.2015.04.002

96. Dutzan N, Konkel JE, Greenwell-Wild T, Moutsopoulos NM. Characterization of the Human Immune Cell Network at the Gingival Barrier. Mucosal Immunol (2016) 9:1163-72. doi: 10.1038/mi.2015.136

97. Delima AJ, Van Dyke TE. Origin and Function of the Cellular Components in Gingival Crevice Fluid. Periodontol (2003) 31:55-76. doi: 10.1034/j.16000757.2003.03105.x

98. Landzberg M, Doering H, Aboodi GM, Tenenbaum HC, Glogauer M. Quantifying Oral Inflammatory Load: Oral Neutrophil Counts in Periodontal Health and Disease. J Periodontal Res (2015) 50(3):330-6. doi: 10.1111/jre.12211

99. Rijkschroeff P, Loos BG, Nicu EA. Impaired Polymorphonuclear Neutrophils in the Oral Cavity of Edentulous Individuals. Eur J Oral Sci (2017) 125(5):371-8. doi: 10.1111/eos.12367

100. Rijkschroeff P, Loos BG, Nicu EA. Oral Polymorphonuclear Neutrophil Contributes to Oral Health. Curr Oral Health Rep (2018) 5:211-20. doi: 10.1007/s40496-018-0199-6

101. Jones MM, Vanyo ST, Ibraheem W, Maddi A, Visser MB. Treponema Denticola Stimulates Oncostatin M Cytokine Release and De Novo Synthesis in Neutrophils and Macrophages. J Leukocyte Biol (2020) 108(5):1527-41. doi: 10.1002/JLB.4MA0620-072RR

102. Lakschevitz FS, Aboodi GM, Glogauer M. Oral Neutrophils Display a SiteSpecific Phenotype Characterized by Expression of T-Cell Receptors. J Periodontol (2013) 84(10):1493-503. doi: 10.1902/jop.2012.120477

103. Rijkschroeff P, Jansen ID, van der Weijden FA, Keijser BJ, Loos BG, Nicu EA. Oral Polymorphonuclear Neutrophil Characteristics in Relation to Oral Health: A Cross-Sectional, Observational Clinical Study. Int J Oral Sci (2016) 8:191-8. doi: 10.1038/ijos.2016.23

104. Fridlender ZG, Sun J, Mishalian I, Singhal S, Cheng G, Kapoor V, et al. Transcriptomic Analysis Comparing Tumor-Associated Neutrophils With Granulocytic Myeloid-Derived Suppressor Cells and Normal Neutrophils. PloS One (2012) 7:e31524. doi: 10.1371/journal.pone.0031524

105. Zhang X, Zhang W, Yuan X, Fu M, Qian H, Xu W. Neutrophils in Cancer Development and Progression: Roles, Mechanisms, and Implications (Review). Int J Oncol (2016) 49(3):857-67. doi: 10.3892/ijo.2016.3616

106. Fridlender ZG, Sun J, Kim S, Kapoor V, Cheng G, Ling L, et al. Polarization of Tumor-Associated Neutrophil Phenotype by TGF- $\beta$ : "N1" Versus "N2" TAN. Cancer Cell (2009) 16(3):183-94. doi: 10.1016/j.ccr.2009.06.017

107. Yu T, Tang Q, Chen X, Fan W, Zhou Z, Huang W, et al. TGF- $\beta 1$ and IL-17A Comediate the Protumor Phenotype of Neutrophils to Regulate the Epithelial-Mesenchymal Transition in Oral Squamous Cell Carcinoma. J Oral Pathol Med (2021) 50(94):383-61. doi: 10.1111/jop.13122
108. Domnich M, Riedesel J, Pylaeva E, Kurten CHL, Buer J, Lang S, et al. Oral Neutrophils: Underestimated Players in Oral Cancer. Front Immunol (2020) 11:565683. doi: 10.3389/fimmu.2020.565683

109. Ueta E, Osaki T, Yoneda K, Yamamoto T, Umazume M. Influence of Inductive Chemoradiotherapy on Salivary Polymorphonuclear Leukocyte (SPMN) Functions in Oral Cancer. J Oral Pathol Med (1994) 23:418-22. doi: 10.1111/j.1600-0714.1994.tb00088.x

110. Carmona-Rivera C, Kaplan MJ. Low-Density Granulocytes: A Distinct Class of Neutrophils in Systemic Autoimmunity. Semin Immunopathol (2013) 35 (4):455-63. doi: 10.1007/s00281-013-0375-7

111. Condamine T, Dominguez GA, Youn JI, Kossenkov AV, Mony S, AliceaTorres K, et al. Lectin-Type Oxidized LDL Receptor-1 Distinguishes Population of Human Polymorphonuclear Myeloid-Derived Suppressor Cells in Cancer Patients. Sci Immunol (2016) 1(2):aaf8943. doi: 10.1126/ sciimmunol.aaf8943

112. Marini O, Costa S, Bevilacqua D, Calzetti F, Tamassia N, Spina C, et al. Mature CD10+ and Immature CD10- Neutrophils Present in G-CSF-Treated Donors Display Opposite Effects on T Cells. Blood (2017) 129(10):1343-56. doi: 10.1182/blood-2016-04-713206

113. Garley M, Jablonska E, Dziemianczyk-Pakiela D, Miltyk W, RatajczakWrona W, Borys J, et al. LDGs Versus NDGs in Patients With Oral Squamous Cell Carcinoma (OSCC). Cytokine (2021) 137:155311. doi: 10.1016/j.cyto.2020.155311

114. Sagiv JY, Michaeli J, Assi S, Mishalian I, Kisos H, Levy L, et al. Phenotypic Diversity and Plasticity in Circulating Neutrophil Subpopulations in Cancer. Cell Rep (2015) 10(4):562-73. doi: 10.1016/j.celrep.2014.12.039

115. Bender JS, Thang H, Glogauer M. Novel Rinse Assay for the Quantification of Oral Neutrophils and the Monitoring of Chronic Periodontal Disease. J Periodontal Res (2006) 41:214-20. doi: 10.1111/j.1600-0765.2005.00861.x

116. Silva LM, Brenchley L, Moutsopoulos NM. Primary Immunodeficiencies Reveal the Essential Role of Tissue Neutrophils in Periodontitis. Immunol Rev (2019) 287:226-35. doi: 10.1111/imr.12724

117. Harris ES, Weyrich AS, Zimmerman GA. Lessons From Rare Maladies: Leukocyte Adhesion Deficiency Syndromes. Curr Opin Hematol (2013) 20:16-25. doi: 10.1097/MOH.0b013e32835a0091

118. Visser MB, Sun CX, Koh A, Ellen RP, Glogauer M. Treponema Denticola Major Outer Sheath Protein Impairs the Cellular Phosphoinositide Balance That Regulates Neutrophil Chemotaxis. PloS One (2013) 8:e66209. doi: 10.1371/journal.pone.0066209

119. Uriarte SM, Edmisson JS, Jimenez-Flores E. Human Neutrophils and Oral Microbiota: A Constant Tug-of-War Between a Harmonious and a Discordant Coexistence. Immunol Rev (2016) 273:282-98. doi: 10.1111/ imr.12451

120. Van Dyke TE, Warbington M, Gardner M, Offenbacher S. Neutrophil Surface Protein Markers as Indicators of Defective Chemotaxis in LJP. J Periodontol (1990) 61:180-4. doi: 10.1902/jop.1990.61.3.180

121. Nemoto E, Nakamura M, Shoji S, Horiuchi H. Circulating Promyelocytes and Low Levels of CD16 Expression on Polymorphonuclear Leukocytes Accompany Early-Onset Periodontitis. Infect Immun (1997) 65:3906-12. doi: 10.1128/iai.65.9.3906-3912.1997

122. Roberts HM, Ling MR, Insall R, Kalna G, Spengler J, Grant MM, et al. Impaired Neutrophil Directional Chemotactic Accuracy in Chronic Periodontitis Patients. J Clin Periodontol (2015) 42:1-11. doi: 10.1111/jcpe.12326

123. Nicu EA, Rijkschroeff P, Wartewig E, Nazmi K, Loos BG. Characterization of Oral Polymorphonuclear Neutrophils in Periodontitis Patients: A CaseControl Study. BMC Oral Health (2018) 18:149. doi: 10.1186/s12903-0180615-2

124. Lakschevitz FS, Hassanpour S, Rubin A, Fine N, Sun C, Glogauer M. Identification of Neutrophil Surface Marker Changes in Health and Inflammation Using High-Throughput Screening Flow Cytometry. Exp Cell Res (2016) 342:200-9. doi: 10.1016/j.yexcr.2016.03.007

125. Beyrau M, Bodkin JV, Nourshargh S. Neutrophil Heterogeneity in Health and Disease: A Revitalized Avenue in Inflammation and Immunity. Open Biol (2012) 2:120134. doi: 10.1098/rsob.120134

126. Magnusson I, Marks RG, Clark WB, Walker CB, Low SB, Mcarthur WP. Clinical, Microbiological and Immunological Characteristics of Subjects With "Refractory" Periodontal Disease. J Clin Periodontol (1991) 18 (5):291-9. doi: 10.1111/j.1600-051X.1991.tb00431.x 
127. Aboodi GM, Goldberg MB, Glogauer M. Refractory Periodontitis Population Characterized by a Hyperactive Oral Neutrophil Phenotype. J Periodontol (2011) 82(5):726-33. doi: 10.1902/jop.2010.100508

128. Chapple ILC, Matthews JB. The Role of Reactive Oxygen and Antioxidant Species in Periodontal Tissue Destruction. Periodontol 200 (2007) 43:160232. doi: 10.1111/j.1600-0757.2006.00178.x

129. Stroncek DF, Caruccio L, Bettinotti M. CD177: A Member of the Ly-6 Gene Superfamily Involved With Neutrophil Proliferation and Polycythemia Vera. J Transl Med (2004) 2(1):8. doi: 10.1186/1479-5876-2-8

130. Goldschmeding R, Van Dalen CM, Faber N, Calafat J, Huizinga TWJ, van der Schoot CE, et al. Further Characterization of the NB 1 Antigen as a Variably Expressed 56-62 kD GPI-Linked Glycoprotein of Plasma Membranes and Specific Granules of Neutrophils. Br J Haematol (1992) 81(3):336-45. doi: 10.1111/j.1365-2141.1992.tb08237.x

131. Matsuo K, Lin A, Procter JL, Clement L, Stroncek D. Variations in the Expression of Granulocyte Antigen NB1. Transfusion (2000) 40(6):654-62. doi: 10.1046/j.1537-2995.2000.40060654.x

132. Newman PJ, Berndt MC, Gorski J, White GC, Lyman S, Paddock C, et al. PECAM-1 (CD31) Cloning and Relation to Adhesion Molecules of the Immunoglobulin Gene Superfamily. Science (1990) 247(4947):1219-22. doi: 10.1126/science.1690453

133. Dahlstrand Rudin A, Amirbeagi F, Davidsson L, Khamzeh A, Thorbert Mros S, Thulin P, et al. The Neutrophil Subset Defined by CD177 Expression Is
Preferentially Recruited to Gingival Crevicular Fluid in Periodontitis. J Leukocyte Biol (2021) 109(2):349-62. doi: 10.1002/JLB.3A0520-081RR

134. Fine N, Chadwick JW, Sun C, Parbhakar KK, Khoury N, Barbour A, et al. Periodontal Inflammation Primes the Systemic Innate Immune Response. J Dent Res (2021) 100:318-25. doi: 10.1177/0022034520963710

Conflict of Interest: The authors declare that the research was conducted in the absence of any commercial or financial relationships that could be construed as a potential conflict of interest.

Publisher's Note: All claims expressed in this article are solely those of the authors and do not necessarily represent those of their affiliated organizations, or those of the publisher, the editors and the reviewers. Any product that may be evaluated in this article, or claim that may be made by its manufacturer, is not guaranteed or endorsed by the publisher.

Copyright () 2022 Metcalfe, Anselmi, Escobar, Visser and Kay. This is an open-access article distributed under the terms of the Creative Commons Attribution License (CC BY). The use, distribution or reproduction in other forums is permitted, provided the original author(s) and the copyright owner(s) are credited and that the original publication in this journal is cited, in accordance with accepted academic practice. No use, distribution or reproduction is permitted which does not comply with these terms. 